\title{
Novel radiation hard microfabricated scintillation detectors with high spatial resolution
}

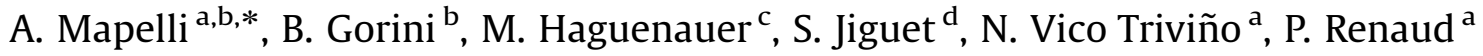 \\ a Laboratoire de Microsystèmes, Ecole Polytechnique Fédérale de Lausanne, Switzerland \\ ${ }^{\mathrm{b}}$ PH Department, CERN, Geneva, Switzerland \\ ${ }^{\mathrm{c}}$ Ecole Polytechnique, CNRS/IN2P3, Palaiseau, France \\ ${ }^{\mathrm{d}}$ Gersteltec Sàrl, Pully, Switzerland
}

\section{A R T I C L E I N F O}

\section{Available online 4 July 2009}

\section{Keywords:}

Scintillation detectors

Microfabrication

Microfluidics

\begin{abstract}
A B S T R A C T
A novel liquid scintillation detector with high spatial resolution is being developed with standard microfabrication techniques. It consists of a dense array of scintillating waveguides obtained by filling microfluidic channels with an organic liquid scintillator and optically coupled to a pixellated photodetector. Such a microfluidic device can be designed and processed to meet the requirements of a wide range of applications like medical imaging, homeland security and high-energy physics. Highspatial resolution miniaturized detectors as well as large-area detectors can easily be fabricated. The fabrication process of a prototype detector and experimental results are presented in this paper.
\end{abstract}

(c) 2009 Elsevier B.V. All rights reserved.

\section{Introduction}

Previous studies have shown that detectors based on capillary bundles filled with liquid scintillators are quite competitive with respect to plastic scintillating fibres devices [1]. They consist of low refractive index capillaries filled with a higher refractive index liquid scintillator to produce a waveguide. Liquid scintillators are fast, efficient and exhibit a good radiation resistance which makes them particularly interesting for particle tracking [2] and calorimetric detectors [3] in high energy physics experiments. The construction of these detectors was reported to be the following. It started with the production of macro-capillaries of borosilicate glass with a diameter of the order of $1 \mathrm{~mm}$. Many capillaries were packed together and drawn at high temperature to obtain a coherent array of micro-capillaries fused together. Several arrays were assembled together and drawn once more to give the final bundles with capillary diameter of the order of $20-30 \mu \mathrm{m}$. Capillary layers could then be constructed by aligning and gluing the bundles while trying to minimize the dead space in between. Finally the layers were cut for a good optical contact to the photodetector window and the capillaries were filled with a liquid scintillator. Even though good experimental results have been obtained with such capillary detectors [1-3] their implementation was rather complicated and many imperfections could arise during each step of their fabrication process.

\footnotetext{
* Corresponding author at: Laboratoire de Microsystèmes, Ecole Polytechnique Fédérale de Lausanne, Switzerland.

E-mail address: alessandro.mapelli@epfl.ch (A. Mapelli).
}

This paper reports on the development of a new type of liquid scintillation detector where an individual capillary layer is obtained by a single step of photolithography guaranteeing a perfect alignment and a regular pitch of the capillaries. This novel detector consists on the assembly of a simple microfluidic chip containing liquid scintillators with a pixellated photodetector.

\section{Microfluidic scintillation detector}

The detector is based on a simple microfluidic device designed to define a dense array of optically separated scintillating waveguides. It is fabricated by photostructuration of the radiation hard epoxy resin SU-8 [4,5] deposited with a controlled thickness on a silicon substrate and coupled to a multi-anode photomultiplier tube (MAPMT). The SU-8 photoresist is commonly used for MEMS [6] and microfluidic devices [7] but also for the fabrication of micro-pattern gas detectors [8] and X-ray imagers [9]. The microfluidic device is made of a single channel with a serpentine geometry. The operation of such a device is very simple as it has only one inlet and one outlet. Once filled with liquid scintillator it comes to be a densely packed array of capillaries, or waveguides, separated by thin structures defining a high spatial resolution detector.

The fabrication process of the microfluidic chips is fairly simple. It begins with the coating of a silicon substrate with the SU-8 photoresist. The thickness of this SU-8 layer is controlled by the viscosity of the resist, the speed and time of rotation during the spin-coating. Uniform thicknesses of $200 \mu \mathrm{m}$ have been achieved in a single spinning step with the SU-8 resin GM1075 
provided by Gerstelec. It is then exposed to UV radiation through a mask with the desired design. The SU- 8 resin being a negative type photoresist, the exposed parts polymerize whereas there is no cross-linking in the non-exposed parts. After a thermal treatment the non-polymerized resin is dissolved in a solvent revealing the desired polymerized structures. In order to avoid optical cross-talk between channels and to increase light transmission along the channels the SU-8 resin is Au-coated before dicing the wafer to separate individual chips. The design was such that 16 chips $(15 \mathrm{~mm} \times 20 \mathrm{~mm})$ were obtained from a $100 \mathrm{~mm}$ diameter silicon wafer.

The various parameters of the fabrication process of the microfluidic chip have been optimized in order to obtain a dense array of thin walls of the order of $10 \mu \mathrm{m}$ with high aspect ratios of about 1:20 over lengths up to $10 \mathrm{~mm}$. These $200 \mu \mathrm{m}$ high structures are separated by only $50 \mu \mathrm{m}$ gaps which makes the fabrication process and in particular the development step rather challenging. The gap between the walls defines the actual size of the channel to be filled with liquid scintillator. Microfluidic chips with rectangular waveguides $50 \mu \mathrm{m}$ wide and $200 \mu \mathrm{m}$ high separated by $10 \mu \mathrm{m}$ wide straight walls were obtained.

\section{Experimental set-up}

To demonstrate the principle of operation of this new type of scintillation detector a microfluidic chip was optically coupled to a pixellated photodetector and tested in a laboratory set-up by exposing the liquid scintillator to $\beta^{-}$particles from a radioactive source. Each waveguide was coupled to one of the pixels of an MAPMT (H7546B by Hamamatsu). They were closed by a thin foil of Al-coated Mylar and encapsulated in a light tight plexiglas container. They were filled with the liquid scintillator EJ-305 by Eljen Technology exhibiting a high light output (80\% of Anthracene). Electrons from a ${ }^{90} \mathrm{Sr}$ source, considered as MIPs, excited the liquid scintillator over the full depth of the channels $(200 \mu \mathrm{m})$. A small fraction of the scintillation light produced by their interaction with the liquid scintillator was guided inside the microchannel and detected by the corresponding pixel of the MAPMT. Its read-out was triggered by the coincidence of the signals from two scintillating fibres aligned with the liquid scintillator waveguides and placed behind the detector along the particle path.

\section{Results and discussion}

The photoelectric yield of the assembly was measured to be of the order of 1 photoelectron per MIP for individual waveguides. A typical charge spectrum is presented in Fig. 1. The pedestal is fitted with a Gaussian and the detector response is fitted with the sum of Poissonians convoluted with Gaussians. Even though the absolute value of this result may seem quite low it is in full agreement with the expectations from theoretical considerations of the microchannel-MAPMT assembly [10]. One of the main factors leading to this rather poor photoelectric yield is the low number of scintillation photons produced along the relatively short path of interaction $(200 \mu \mathrm{m})$ in a single waveguide. An important fraction of these photons are lost before reaching the

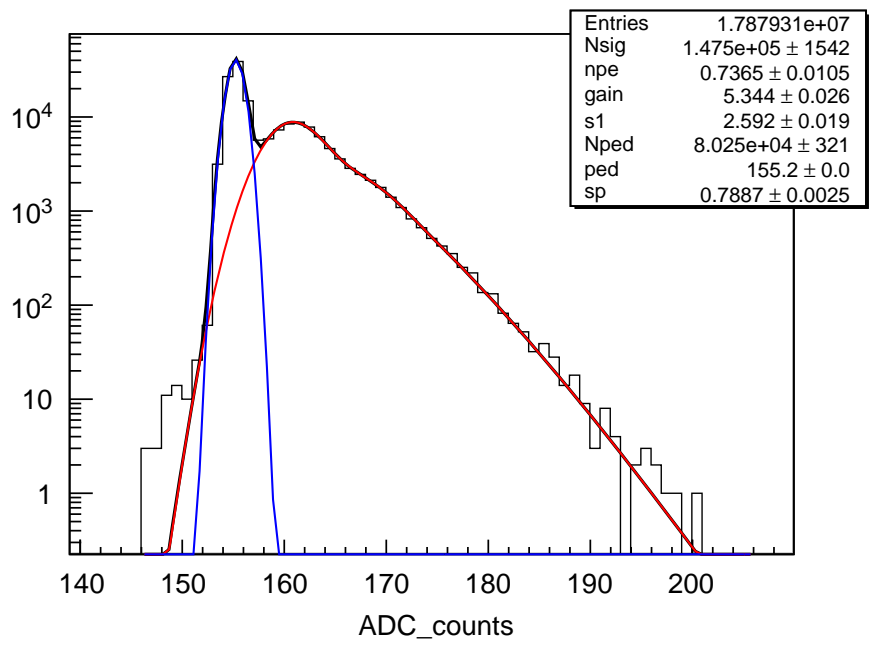

Fig. 1. Response spectrum of a single channel of the microfluidic scintillation detector.

photocathode because of a non-optimized design of the microchannel in terms of waveguiding properties. Moreover it is quite difficult to guarantee a perfect optical contact between the thin detector and the flat window of the MAPMT. A new series of chips have been produced with improved waveguiding properties of the microfluidic channels. They are expected to give a better photoelectric yield under the same experimental conditions.

\section{Conclusions}

By optimizing the parameters of a standard microfabrication process with a radiation hard photoresist it was possible to develop a thin microfluidic device with high spatial resolution and low material budget. Easy manipulation of liquid scintillators by microfluidic techniques in this device allow their flushing and renewal making the active medium intrinsically radiation hard. Moreover the possibility to change the type of liquid scintillator circulating in the device allows to use it over a large energy range and for different types of particles. The experimental results obtained with this scintillating microfluidic device demonstrate a new detection principle that could be of interest for high resolution particle tracking in high radiation environments.

\section{References}

[1] A. Bay, et al., Nucl. Instr. and Meth. A 457 (2001) 107.

[2] S. Buontempo, et al., Nucl. Instr. and Meth. A 360 (1995) 7.

[3] A. Artamov, et al., Nucl. Instr. and Meth. A 360 (1995) 240.

[4] H. Lorenz, et al., J. Micromech. Microeng. 7 (1997) 121.

[5] M.J. Key, et al., Rad. Phys. Chem. 71 (2004) 1003.

[6] H. Lorenz, et al., Sens. Act. A 64 (1998) 33.

[7] S. Metz, et al., Lab Chip 4 (2004) 114.

[8] J. Schmitz, Nucl. Instr. and Meth. A 576 (2007) 142.

[9] J.H. Daniel, et al., Sens. Act. A 140 (2007) 185.

[10] A. Mapelli, et al., to appear in Nucl. Phys. B, in: Proceedings of Supplementary for the IPRD08 Conference in Siena, Italy, 2008. 